\author{
AMR HELMY IBRAHIM \\ Cellule de Recherche Fondamentale en Linguistique Française \\ et Comparée Université de Franche-Comté (Besançon)
}

\title{
RÉPÉTITION, REDONDANCE, REFORMULATION: LES SPIRALES EXPLICATIVES DU MÊME
}

\begin{abstract}
A bstract. Ibrahim Amr Helmy, Répétition, redondance, reformulation: les spirales explicatives du même [Repetition, redundance, rewording: the spiral explaining the same]. Studia Romanica Posnaniensia, Adam Mickiewicz University Press, Poznań, vol. XXV/XXVI: 2000, pp. 157-166, ISBN 83-2320965-0, ISSN 0137-2475.

This work tries to show how and why repetition, redundancy and rewording, by borrowing the figure of some sort of a spiral do explain and add considerably to meaning in spite of the fact that they don't seem to be willing to do so. Although only redundancy is likely to occur with the same shape in all languages, the three are indeed universals even if their function and place in the grammar is not the same in all languages. The paper refers to three types of ongoing different researches: that of the followers of Zellig $\mathrm{S}$. Harris about redundancy seen as a complete descriptive tool of language, that of the author about repetitions in the holy Coran, and that of Claire Martinot about the way children appropriate meaning through rewordings.
\end{abstract}

Le travail dont je présente ici quelques aspects de manière cursive et simplifiée est une contribution modeste à un phénomène particulier de l'analyse des discours. Il n'en a pas moins la prétention d'instituer à terme une nouvelle approche du texte centrée tout à la fois sur la syntaxe et ses mécanismes ponctuels, souvent idiosyncrasiques, parfois mêmes atomiques et sur les conditions effectives de construction du sens à travers un usage de ces textes dont on n'exclut a priori aucun paramètre. Il est fortement tributaire de trois axes de recherche qui n'avaient au départ ni la même motivation ni le même auteur et ne se sont développés, dans un premier temps, ni au même rythme ni avec le même horizon. Il s'agit, dans un ordre qui n'est pas vraiment chronologique, d'une réflexion sur la fonction et la traduction des répétitions dans le Coran $^{1}$, de l'élaboration d'une théorie générale du langage fondée sur des formes

1 Fonction et traduction des répétitions dans le Coran: modulation du même versus dissimilation dans la construction du sens, in: Sur la traduction, Annales de l'Université de Besançon, 1998, 30 p. 
particulières de redondance ${ }^{2}$, et d'une recherche sur la fonction des reformulations dans l'acquisition de la langue maternelle ${ }^{3}$ telles qu'elles ont été observées, définies et analysées pour la première fois par Claire Martinot chez des enfants au cours de pratiques orales interactives et que nous définissons comme étant une remontée vers un sens dont l'existence et la forme font partie des évidences qu'on désire s'approprier.

De ces trois mécanismes universels: répétition, redondance et reformulation, seul le second devrait être transposable sans modification de nature ou de forme dans toutes les langues du monde. Si toutes les langues connaissent la répétition et la reformulation, elles ne leur assignent pas les mêmes fonctions et ne les font pas porter sur les mêmes objets. Répétition et reformulation sont effet fortement dépendants de la grammaire tandis que la redondance produit, à l'intérieur de chaque langue, un sous-ensemble de phrases plus ou moins naturelles mais toujours à la fois explicatives et affranchies d'une grande partie des contraintes de la grammaire. On peut même aller jusqu'à dire que l'ensemble de la grammaire n'est qu'un instrument de cette propension à l'ellipse qui exerce une pression constante sur l'évolution des langues et qu'en reconstruisant ce qui a été élidé, on remonte le chemin par lequel se sont empilées et enchevêtrées les contraintes de la grammaire. Ce caractère explicatif, même très maladroit de la redondance, en fait un arbitre privilégié dans le choix aussi bien des stratégies didactiques que de la bonne traduction.

$\mathrm{Si}$, par exemple, nous cherchons à développer par redondance l'énoncé français

Voler, quel bonheur!

par opposition à l'énoncé italien:

(2) Rubbare, che felicità!

sous les formes:

1a) [Quelqu'un (dit+énonce) + On voit écrit] une suite de mots qui (disent + signifient) que le fait pour quelqu'un de s'élever dans les airs et d'y avancer dans une certaine direction (est la source d' + procure) un sentiment de bonheur.

1b) [Quelqu'un (dit + énonce) + On voit écrit] une suite de mots qui (disent + signifient) que le fait pour quelqu' un qui n'a pas le droit de (faire + prendre) quelque chose de (prendre + faire) cette chose (au détriment + contre le gré) de quelqu'un qui (possède + est maître de l'usage) de cette chose et de garder cette chose pour (lui-même + quelqu'un d'autre que celui à qui il a prise cette chose) (est la source d' + procure) un sentiment de bonheur.

${ }^{2}$ La forme d'une théorie du langage axée sur les termes supports, Langages 121, mars 1996 \& Pour une définition matricielle du lexique, Cahiers de Lexicologie, vol. 70-2, 1997.

${ }^{3}$ C. Mart inot, La reformulation dans des productions orales de définitions et explications (Enfants de classe maternelle). Thèse de doctorat en Sciences du langage soutenue le 15.01.94 à l'Université Paris VIII (Disponible sur microfiches) - Cf. également Reformulations chez de jeunes locuteurs: construction d'un discours à plusieurs voix, Cahiers du français contemporain, 3, juin 1996, p. 175-191. 
induit une définition, une désambiguïsation - l'énoncé italien ne peut recevoir qu'une lecture équivalente à $1 \mathrm{a}$ - un dépliage des paramètres énonciatifs et une neutralisation des contraintes grammaticales. Le tout avec une valeur explicative.

\section{RÉCURRENCE, COHÉSION ET DIFFÉRENCIATION DU SENS}

Les nombreuses approches passées et présentés de l'analyse du discours s'accordent sur au moins un point: la cohésion d'un texte, et singulièrement d'un texte relativement long, est en grande partie et parfois uniquement tributaire d'une forme ou d'une autre de récurrence.

Celle-ci, lorsque l'ensemble des composantes du texte a un fort degré de cohésion ou d'intégration, devrait, comme l'a suggéré Joseph Sumpf, se cristalliser en une phrase unique, une sorte de phrase emblématique du texte qui en résume pour ainsi dire toutes les potentialités. Elle devrait aussi révéler deux caractéristiques qui sont généralement considérées comme appartenant respectivement aux domaines de la stylistique et de la cognition: le ton d'un texte d'une part, la forme d'appropriation dont il est l'objet d'autre part. C'est-à-dire que d'une part elles marquent le texte d'une empreinte qui le singularise ou tout au moins permet de le reconnaître comme produit par un individu ou un type d'individus assignable, d'autre part elles favorisent son appropriation comme instrument de compréhension et de découverte, de révélation du monde.

Pour comprendre comment et pourquoi la récurrence produit cohésion, cristallisation, tonalité singulière et enfin révélation, il convient d'abord d'examiner la nature des récurrences et leur typologie.

La répétition d'un élément de la langue est susceptible d'entraîner un changement de sa signification ou tout au moins de la fonction qu'il assure dans la construction du sens. Les énoncés:

1) Qu'est-ce qu'on dit quand on dit ce que tu dis?

ou

1') Il y a des linguistes qui s'attachent à montrer ce que l'on dit en disant ce que l'on dit

peuvent recevoir assez naturellement les paraphrases suivantes:

1a) Qu'est-ce qu'on veut dire quand on énonce ce que tu dis

1'a) Il y a des linguistes qui s'attachent à montrer ce que l'on veut dire en énonçant ce que l'on dit.

1b) Qu'est-ce qu'on entend par l'énonciation de ce que (tu viens de dire + tu as l'habitude de dire)

l'b) Il y a des linguistes qui s'attachent à montrer ce que l'on a entendu en énonçant ce que l'on dit.

1c) Qu'est-ce que l'on signifie quand on énonce ce que l'on dit

l'c) Il y a des linguistes qui s'attachent à montrer ce que l'on signifie en énonçant ce que l'on dit. 
Autrement dit, la $1^{\text {ère }}$ occurrence de dire en fait un quasi synonyme de signifier, la deuxième du processus de production de ce qui est dit, son énonciation, tandis que la troisième, apparemment la plus neutre, est la seule à ne pas varier au cours des paraphrases. Elle semble n'être que ce qui est dit, le résultat linguistique du fait de dire.

L'interprétation change un peu si on remplace dire par manger mais les valeurs différentielles restent les mêmes:

2) (Qu'est-ce qu'on mange quand on mange ce que tu manges? + Est-ce que tu te rends compte de ce que tu manges en mangeant ce que tu manges?)

2a) ((Quelle est + Est-ce que tu te rends compte de) la nature de ce que $(l ' o n+t u)$ mange $(s)$ $(((q u a n d$ on (avale + ingurgite $))+($ en (avalant + ingurgitant $)))$ ce que tu manges.

La première occurrence renvoie toujours à la valeur ou à la nature de l'acte, la deuxième au fait même de faire ce que l'on fait, et la troisième à la matérialité du fait.

On peut se livrer à ce petit jeu avec pratiquement n'importe quel verbe, y compris avec les verbes intransitifs:

3) Comment dort-on quand on dort comme tu dors?

3a) Quelle est (la nature + la forme) du (sommeil + dormir) quand on dort de (la façon + manière) dont tu dors?

La seule différence entre (3) et (2) ou (1) c'est que la $3^{\text {ème }}$ occurrence n'est pas un résultat neutre de l'action exprimée dans la $2^{\text {ème }}$ occurrence mais un état particulier, une forme spécifique de réalisation de cette action.

Ainsi, pour dire, la première forme est une métonymie: vouloir dire pour dire, la deuxième un autonyme: le fait de dire pour dire, et la troisième la dénomination neutre ou qualifiée de ce qui est dit ou de la façon de le dire.

Ces trois phases du même correspondent à trois manières distinctes par lesquelles tout discours appréhende une réalité lexicale:

(1) Il l'instrumentalise: un mot pour un autre. C'est aussi une forme de déplacement.

(2) Il la réfléchit: il pointe le fait même d'user de ce mot là et pas d'un autre. C'est aussi une mise en perspective.

(3) Il en fait une étiquette. C'est aussi, naturellement, une forme de classement.

Ces trois phases correspondent à des opérations extrêmement générales: déplacer et manipuler l'objet, se situer par rapport à lui, l'étiqueter ou le nommer.

Le fait qu'une répétition puisse s'inscrire dans trois plans différents de signification peut entraîner des dissimilations très nettes malgré une répétition à l'identique du signifiant. Il est en effet tout à fait possible que l'un des trois plans - et c'est souvent le cas en français, dont on a souligné depuis le XVII siècle qu'il détestait les répétitions, génère une signification très différente des deux autres ou tout au moins du plan qui le précède ou le suit: 
Je dis en disant ce que je dis que les lois de ce pays sont injustes

Je mange la chair du Christ en mangeant ce que je mange.

Je ne dors pas, en dormant comme je dors: je me protège.

Mais les chansons de Jacques Brêl seront plus parlantes. D'autant qu'il lui arrive d'y employer une métalangue entièrement et superbement inscrite, selon l'expression de Zellig Sabbetai Harris, dans la langue comme dans Orly:

«...et brusquement il pleure, il pleure à gros bouillons (....) et maintenant ils pleurent, je veux dire tous les deux, tout à l'heure c'était lui lorsque je disais il’

ou, toujours dans la même chanson, cette modulation de la répétition, d'autant plus pure que son objet a été effacé:

Ils sont en train de ne rien se promettre

ou encore, dans Voir un ami pleurer ce jeu de renvois qui fait, avec la répétition, du métalangage sans la lourdeur de la métalangue:

Bien sûr l'argent n'a pas d'odeur mais pas d'odeur monte au nez

et enfin, montrant cette sûreté de compréhension de la langue qu'on entrevoyait dans cette remarque des Vieux :

Mourir sous le manteau,

anonyme, incognito,

Mourir comme meurt un synonyme.

ce chef d'œuvre de variations à partir des ressources de la répétition que l'on trouve dans La ville s'endormait. Partant d'une comparaison/répétition purement redondante et dont la symétrie quasi parfaite est comme confirmée par:

Il est vrai que parfois, près du soir

Les oiseaux ressemblent à des vagues

Et les vagues aux oiseaux

il engage une comparaison construite sur une relation transitive qui esquisse un syllogisme:

Et les hommes aux rires

Et les rires aux sanglots

pour introduire la figure majeure de la répétition, cette fois-ci dissimilatrice (les femmes), à travers une homonymie ambiguë (mer/mère), signalée par un jeu de mots (désenchantelchante):

Il est vrai que souvent la mer se désenchante

Je veux dire en cela qu'elle chante d'autres chants

Que ceux que la mère chante

Dans les livres d'enfants

Mais les femmes toujours ne ressemblent qu'aux femmes

Et d'entre elles les connes ne ressemblent qu'aux connes

Et je ne suis pas bien sûr comme chante-t-un certain qu'elles soient l'avenir de l'homme. 
Un exercice dont la signification est confirmée par une violente rupture d'isotopie sur laquelle s'achève brusquement la chanson:

Et vous qui êtes passée, demoiselle inconnue

à deux doigts d'être nue

Sous le lin qui dansait.

\section{LES DIFFÉRENTES VALEURS DE LA RÉPÉTITION}

En fait, toute répétition, même partielle, d'un signifiant, produit, c'est presque un truisme, un effet anaphorique plus ou moins appuyé. Mais elle entraîne également, du fait des contraintes conjuguées de la linéarité des langues et de leur inscription dans une temporalité dont les paramètres sont tributaires de la mémoire des signifiants accumulés, un effet de différenciation comparable à celui que l'on peut trouver dans des coordinations ${ }^{4}$. La pondération de ces deux tendances opposées varie d'une langue à l'autre et présente des caractéristiques bien accusées lorsque l'on compare des langues, même proches, qui n'usent pas exactement du mểme arsenal morpho-syntaxique pour exprimer les différentes formes de reprise. On peut se donner, à partir du français, une première grille sommaire d'évaluation et la rapporter en contexte aux trois valeurs: instrumentalisation/déplacement, éconciation/réflexion, étiquetage/classement que nous avons proposée plus haut:

(1) Possibilité de répéter purement et simplement sans aucune variation la totalité du signifiant, le plus souvent pour indiquer soit la répétition ininterrompue et à l'identique d'un mouvement ou d'un geste, soit pour figer un état ou indiquer une action consacrée institutionnellement et lingustiquement: un pousse-pousse, courir pour courir, vivre pour vivre, coup sur coup, face à face, tête à tête, main dans la main, bras dessus bras dessous, Quand je dis je ne veux pas c'est que je ne veux pas, mais aussi ces propos recueillis dans le RER à Paris: «Tu sais, les enfants y pensent pas comme nous, hein, quand y z'ont pas envie, y z'ont pas envie».

(2) Possibilité de reprendre le signifiant avec des variations morpho-phonématiques mineures qui contrastent avec un effet sémantique majeur: une politique politicienne, enfin la fin!, parisien peut-être pharisien certainement. Et plus largement: Ils ont changé ma chanson pour Ils ont chanté ma chanson.

(3) Possibilité de substituer à la reprise une pronominalisation complète de substitution: ton livre je l'achète; une substitution générique: ton livre il s'en écrit dix tous les jours de par le monde, ou, avec l'ambiguïté sur le type ou la référence individualisante: ton livre j'en achèterai trois; une substitution de partition: ton livre, j'en ai lu un chapitre, cette soupe j'en reprendrais bien.

(4) Possibilité de préciser ou de moduler la reprise par un ensemble de déterminants et/ou de déictiques dont l'usage s'organise autour de systèmes plus ou moins complexes d'oppositions signifiantes: ce sang qu'il lui a transfusé, c'était son sang, sa vie.

\footnotetext{
${ }^{4}$ Amr Helmy Ibrahi m, 1978, Coordonner pour argumenter, Semantikos, vol. 2, n 2-3, Paris.
} 
(5) Possibilité de jouer sur l'ordre des mots du groupe repris: c'est bonnet blanc, blanc bonnet.

(6) Possibilité de produire des effets quantitatifs ou des valeurs modales: Elle est belle, belle, comme le jour, C'est une loco, loco, locomo, locomo, locomotive d'or...., pas à pas, petit à petit, peu à peu, les prendre un à un, faire du porte à porte. Soit dit en passant, une comptine italienne combine ce procédé à une contradiction référentielle pour développer une critique sociale: $i$ pappaveri son alti, alti, alti $e$ tu sei piccolina, e tu sei piccolina... (la répétition de alti (hauts) attire l'attention sur une contradiction du fait que la plante du pavot n'est pas «très haute» et oriente vers son sens figuré sous-entendu: grosses légumes au sens figuré français).

(7) Existence d'affixes ayant parmi leurs fonctions celle d'une reprise unaire ou générique comme bi-, tri-, poly-, re-, -eur, -able, -iste, -iller.

Toutes ces formes de répétitions ont donc on le voit une caractéristique commune: elles sont, du fait de la redondance plus ou moins importante qu'elles génèrent, $e x$ plicatives, ou tout au moins définitoires. Or, la définition comme l'explication se réalisent lorsqu'elles sont explicites sous trois formes majeures bien distinctes mais nécessairement reliées en dernière analyse:

(1) La synonymie ou l'équivalence par le biais d'une paraphrase sémantique, d'une transformation syntaxique ou d'une restructuration. Nous n'en parlerons pas ici.

(2) L'équivalence par redondance.

(3) La reformulation.

La répétition est quant à elle la forme implicite de la définition ou de l'explication.

\section{LA REDONDANCE COMME PRINCIPE DE DÉFINITION ANALYTIQUE}

\section{Brêl chante dans Orly:}

Ils se tenaient par les yeux

On peut comprendre cet énoncé comme étant la réduction par une suite d'effacements réglés ${ }^{5}$ de la suite suivante:

A un moment que je vous présente comme antérieur au moment où vous m'entendez et pendant un temps que je ne précise pas deux personnes, les yeux, qui sont des parties du corps, orientés par le regard les uns vers les autres comme si l'un allait dans l'autre, se tiennent par le regard que chaque personne porte avec ses yeux sur l'autre comme si ces deux personnes se tenaient par exemple par la main.

${ }^{5}$ La technique des effacements d'éléments redondants a été inaugurée par Zellig Sabbetai Harris. Pour une référence en français on se reportera à Notes du cours de syntaxe, Paris, Seuil, 1974. Nous l'avons développée dans nos analyses matricielles. On pourra ajouter aux références de la note $2 \mathrm{Amr}$ Helmy Ibrahim, Théorie générale: l'articulation de l'arbitraire et du motivé par les individus de la langue, Opérateurs, Supports, Durées, Annales Littéraires de l'Université de Besançon, Série Linguistique et Sémiotique, $n^{\circ} 512$, Paris, Les Belles Lettres. 
Seules sont effaçables les séquences reconstructibles. La validité de cette définition analytique dépend donc de la possibilité de la construire à partir de l'énoncé de départ sans recours à une information étrangère au contexte où cet énoncé a été produit et, plus généralement, - ce n'est pas le cas ici mais c'était le cas dans l'exemple de définition analytique que nous avons donné précédemment - à tous les contextes type où il pourrait être produit. Dans le cas qui nous intéresse les séquences effaçables du fait d'une forme ou d'une autre de redondance portent sur:

1 - Les coordonnées temporelles et aspectuelles.

2 - Les distributions potentielles qu'entraine l'intersection de tenir et par les yeux. Ces distributions sont complexes mais toutes entières contenues dans les valeurs lexicales virtuelles des termes telles qu'elles sont consignées dans un dictionnaire courant de langue. On remonte à regard par les yeux et on établit l'ensemble des actualisateurs sémantiques et grammaticaux liés à ces termes et contraints par le contexte.

On voit facilement les implications didactiques et traductologiques de cette approche: ce par quoi les langues diffèrent le plus est leur grammaire au sens de l'ensemble des contraintes formelles notamment syntaxiques qui commandent la construction du sens. Ces contraintes contiennent probablement la part la plus arbitraire des langues si l'on exclut l'arbitraire premier qui régit la différenciation des signes élémentaires. Or l'approche par la redondance, en dépliant et en explicitant au maximum l'énoncé annule ces contraintes ou leur donne une forme analytique dont les composants sont universels et peuvent avoir une fonction doublement transparente: au regard de l'enseignement et plus largement dans toute démarche didactique et au regard de la traduction. Le dépliement de l'énoncé est, simultanément, la formulation d'une hypothèse sur le cheminement suivi par des composants, élémentaires aussi bien du point de vue du lexique que de la grammaire, pour s'intégrer sous des formes plus synthétiques et plus ramassées qui ne gardent pas forcément, que ce soit dans leur morphologie ou leur sens actuel, la mémoire de leur généalogie. Mais le dépliement ne fournit pas seulement des indices sur l'histoire, au sens chronologique et diachronique, des formes, c'est également un indicateur de leur fonctionnement ici et maintenant et de leurs différentes interprétations possibles en synchronie. Toutes choses dont aucun traducteur sérieux ne peut se passer.

La réalisation du dépliement d'un énoncé par la production de redondances obéit à des principes très simples:

1 - Il faut procéder à toutes les nominalisations possibles et plus généralement retrouver pour tous les termes leurs bases non prédicatives afin de voir sous une forme lexicale élémentaire la forme des éléments qui les actualisent ou encore qui les grammaticalisent.

2 - 11 faut retrouver les réseaux de dérivation et d'association strictement morphologiques et ceci même si la langue décrite n'exploite pas systématiquement et régulièrement, comme le font par exemple nombre de langues romanes, des procédés d'affixation (préfixes, infixes, suffixes), ou, comme le font, par exemple, les langues sémitiques, des procédés de variation de schèmes. 
3 - Il faut développer au maximum la verbalisation de la deixis, c'est-à-dire de tous les éléments qui ont pour fonction de pointer un élément quel qu'il soit à l'intérieur ou à l'extérieur de l'énoncé observé.

\section{L'ÉQUIVALENCE PAR REFORMULATION}

La reformulation telle qu'elle a été définie par Claire Martinot puis développée au sein de la CRFLFC et ailleurs est une forme de modulation fonctionnelle et finalisée de la reprise qui se constitue en instrument de compréhension, d'expression, de découverte ou des trois à la fois. Sa grande richesse vient du fait que répondant à un besoin indiscutable imposé par une situation de communication nettement déséquilibrée - sans d'ailleurs que ce déséquilibre soit forcément connoté négativement - elle construit l'instrument en même temps que les paramètres de l'énonciation et s'adresse alors indifféremment à soi-même ou à autrui ou aux deux. Si cette construction est réussie, c'est-à-dire si l'auteur des reformulations estime que ses reformulations lui ont permis de comprendre, de se faire comprendre ou de découvrir quelque chose, la construction de l'instrument aura triomphé du déséquilibre initial. Autrement dit l'élaboration de l'instrument aboutit à sa propre validation. Peu d'outils linguistiques bénéficient d'une gamme aussi complète de fonctions au sein des phénomènes d'acquisition, de construction et d'expression du sens.

Dans son étude, envisagée au départ comme la reçerche d'instruments majeurs en usage dans les pratiques assistées d'acquisition du sens par des enfants scolarisés, Claire Martinot a dégagé quatre fonctions fondamentales de la reformulation: la dé'finition, l'intensification ou l'insistance, la rectification et l'explication. Ces fonctions sont doublement universelles: culturellement et linguistiquement. Elles sont toutes, au départ, fondées sur une forme ou une autre de répétition et usent d'une forme ou d'une autre de redondance mais la reformulation excède et la répétition et la redondance telles que nous les avons présentées. Ses voies, et il suffit d'observer de près des enfants ou des adultes qui en usent ou d'en suivre les traces dans un texte, peuvent être imprévisibles, tout au moins dans une première approximation. Et la question reste ouverte si ces formes de répétition ou de redondance peuvent être ramenées aux schémas réglés de répétition et de redondance que nous avons étudiés et étudions ou si de nouvelles formes d'explication du mécanisme à l'œuvre sont nécessaires.

\section{CONCLUSION}

Jorge Donn, à la fin du film de Claude Lelouch, Les uns et les autres, danse le Boléro de Maurice Ravel sur une chorégraphie de Maurice Béjart. Des modulations, une variation que l'on voudrait infinie sur un thème récursif mais aussi un renouvel- 
lement permanent dans le cadre d'un éternel retour. Comme si chaque recommencement charriait une nouvelle signification, comme si chaque cercle, un peu plus large que le précédent, allait cueillir une part nouvelle du monde sans que son centre ne se déplace pour autant. Dilatation, expansion diversifiée à perte de vue, de l'un, de l'unique. Spirale dont on jurerait qu'elle se meut mais dont le mouvement, pourtant explicatif, n'est presque, en réalité, comme dans les gravures de Maurits Cornelis Escher, qu'une illusion de mouvement. 